Revista de Investigación de Física 23(3), (2020)

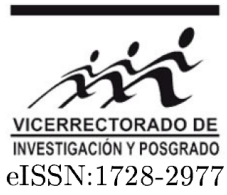

\title{
La concentración de la clorofila-a y su relación con la temperatura superficial del mar, mediante imágenes de satélite y datos in-situ, en las bahías de Sechura y Paita
}

\author{
Gilberto Ramírez $^{1 *}$, Joel Rojas ${ }^{2,3}$ y Carlos Carbonell ${ }^{3}$ \\ ${ }^{1}$ Universidad Nacional San Cristóbal de Huamanga \\ ${ }^{2}$ Universidad Nacional Mayor de San Marcos \\ ${ }^{3}$ Centro de Investigaciones Tecnológicas, Biomédicas y Medio Ambientales (CITBM) \\ Recibido 12 mayo 2020 - Aceptado 11 diciembre 2020
}

\begin{abstract}
Resumen
Se ha realizado la validación de la Temperatura de la Superficie del Mar (TSM) estimada desde imágenes de los sensores MODIS y VIIRS, para el área de estudio delimitados entre las longitudes de $83^{\circ}-80^{\circ} \mathrm{O}$ y latitudes de $3^{\circ}-7^{\circ} \mathrm{S}$ respectivamente, en el periodo de 2001 a 2016 con datos de la TSM in-situ. Asimismo, se ha relacionado los datos promedios mensuales de la Concentración de Clorofila-a (CCA) y TSM VIIRS para la bahía de Sechura, en el periodo de 2012 a 2018. Los datos in-situ de TSM son registrados por IMARPE, (2000-2015). Las imágenes CCA y TSM diarias y mensuales para el área de estudio son obtenidas del portal Ocean Color. Se ha usado el software de procesamiento de imágenes SeaDAS versión 7.5.3 en Linux Ubuntu 16.04 con formato netcdf. Los promedios mensuales de TSM-MODIS y la TSM in-situ en la estación de Paita presenta un coeficiente de correlación $\mathrm{R}=0.96$, por lo que podemos plantear su uso en modelos de variables oceanográficos. Según el promedio climatológico provisional de TSM (2008-2015) para las bahías de Sechura y Paita, los valores de temperatura oscilan entre $19.5^{\circ} \mathrm{C}$ y $22^{\circ} \mathrm{C}$, con desviación típica de $0.26^{\circ} \mathrm{C}$. El histograma de frecuencia de la TSM climatológico para la bahía de Sechura presenta una distribución bimodal sesgada hacia la derecha. El algoritmo Split Window NLSST para MODIS y VIIRS sobreestimaron las medidas in-situ de TSM. La bahía de Sechura presenta una correlación positiva significativa ( $\mathrm{R}=70.4 \%$ ) entre TSM y CCA. Se ha observado que el aumento de la temperatura influye en el incremento de la CCA de la zona.
\end{abstract}

Palabras claves: TSM, CCA, Split Window, MODIS y VIIRS.

\section{The concentration of chlorophyll-a and its relationship with the sea surface temperature, through satellite images and in-situ data, in the bays of Sechura and Paita}

\begin{abstract}
The validation of the Sea Surface Temperature (SST) estimated from images of the MODIS and VIIRS sensors has been carried out, for the study area delimited between the longitudes of $83^{\circ}-80^{\circ} \mathrm{W}$ and latitudes of $3^{\circ}-7^{\circ} \mathrm{S}$ respectively, in the period from 2001 to 2016 with data from the SST in-situ. Likewise, the monthly average data of the Concentration of Chlorophyll-a (CCA) and TSM VIIRS for the Sechura Bay, in the period from 2012 to 2018, have been related. The in-situ data of TSM are registered by IMARPE, (2000 2015). The daily and monthly CCA and TSM images for the study area are obtained from the Ocean Color portal. SeaDAS version 7.5.3 image processing software has been used on Linux Ubuntu 16.04 with netcdf format. The monthly averages of SST-MODIS and the in-situ SST at the Paita station present a correlation coefficient $\mathrm{R}=0.96$, so we can propose its use in models of oceanographic variables. According to the provisional climatological average of SST (2008-2015) for the bays of Sechura and Paita, the temperature values range between $19.5^{\circ} \mathrm{C}$ and $22{ }^{\circ} \mathrm{C}$, with a standard deviation of $0.26{ }^{\circ} \mathrm{C}$. The frequency histogram of the climatological SST for Sechura Bay shows a bimodal distribution skewed to the right. The Split Window NLSST algorithm for MODIS and VIIRS overestimated the in-situ measurements of SST. Sechura Bay presents a significant positive correlation $(\mathrm{R}=70.4 \%)$ between SST and CCA. It has been observed that the increase in temperature influences the increase in the CCA of the area.
\end{abstract}

Keywords: TSM, CCA, Split Window, MODIS and VIIRS.

*cfmgilbertq@gmail.com 
Rev. Inv. Fís. 23(3), (2020)

\section{Introducción}

En este trabajo se utilizaron los productos TSM de nivel L2 y L3 correspondiente a los sensores MODIS y VIIRS a bordo de los satélites Aqua y SUOMI-NPP (Suomi National Polar-Orbiting Partnership) de observaciones diurnas, los cuales son recolectadas desde el portal web OceanColor (http://oceandata.sci.gsfc.nasa.gov/2. Los satélites cruzan el ecuador en nodo ascendente aproximadamente a la 13:30 (hora local). Los datos imágenes diarios de TSM nivel 2 corresponden a MODIS (versión R2014.0) de resolución espacial de $1 \mathrm{~km}$ y a VIIRS (versión R2016.0) de 750 m de resolución espacial. Asimismo, la TSM de nivel 3 usado para la climatología mensual es de tipo SMI (Standard Mapped Image), con resolución espacial de $4 \mathrm{~km}$ y formato NetCDF. El procesamiento y la distribución estándar por la NASA de los productos TSM de los sensores MODIS y VIIRS se realizan utilizando el software desarrollado por el OBPG (Ocean Biology Processing Group). El OBPG genera el producto TSM de nivel 2 utilizando el código l2gen del procesador multilevel instalado en el software SeaDAS, que es el mismo código utilizando para generar productos del color de océano MODIS y VIIRS. El método utilizado para derivar la TSM en el infrarrojo (IR) a partir de mediciones de las temperaturas de brillo en la cima de la atmosfera (TOA) es a través del algoritmo Split Window NLSST (TSM no lineal) propuesto por (Walton et al., 1998), para condiciones de una atmosfera libre de nubes y es expresado por la siguiente ecuación:

$T S M=a_{0}+a_{1} T_{11}+a_{2}\left(T_{11}-T_{22}\right) T_{\mathrm{sfc}}+a_{3}\left(T_{11}-T_{12}\right)(\sec (\theta)-1)$

donde $a_{0}, a_{1}, \mathrm{a}_{2}$ y $a_{3}$ son los coeficientes, $T_{11}$ es la temperatura de brillo medida en la banda centrada cerca de $\lambda=11 \mu \mathrm{m}, T_{12}$ es la temperatura de brillo medida en la banda centrada cerca de $\lambda=12 \mu \mathrm{m}$. $T_{\text {sfc }}$ es una estimación de la temperatura de la superficie y $\theta$ es el ángulo cenital del satélite.

La concentración de la clorofila-a (CCA) por satélite en la superficie del mar es recuperado mediante la combinación de dos algoritmos: el algoritmo empírico OCx de O'Reilly (2000) y el algoritmo de Índice de Color (CI) de Hu y otros (2012). El algoritmo CI es un algoritmo de diferencia de reflectancias de tres bandas que emplea la diferencia entre $\mathrm{R}_{\mathrm{rs}}$ en la banda verde $\mathrm{y}$ una referencia formada linealmente entre $\mathrm{R}_{\mathrm{rs}}$ en las bandas azul y rojo.

$\mathrm{CI}=\mathrm{R}_{\mathrm{rs}}\left(\lambda_{\text {verde }}\right)-\left(\mathrm{R}_{\mathrm{rs}}\left(\lambda_{\text {azul }}\right)+\frac{\mathrm{R}_{\mathrm{rs}}\left(\lambda_{\text {verde }}\right)-\mathrm{R}_{\mathrm{rs}}\left(\lambda_{\text {azul }}\right)}{\mathrm{R}_{\mathrm{rs}}\left(\lambda_{\text {rojo }}\right)-\mathrm{R}_{\mathrm{rs}}\left(\lambda_{\text {azul }}\right)} *\left(\mathrm{R}_{\mathrm{rs}}\left(\lambda_{\text {rojo }}\right)-\mathrm{R}_{\mathrm{rs}}\left(\lambda_{\text {azul }}\right)\right)\right)$ donde $\lambda_{\text {azul }}, \lambda_{\text {verde }} \mathrm{y} \lambda_{\text {rojo }}$ son las longitudes de onda específicas del instrumento más próximas a 443, 555 y 670 $\mathrm{nm}$ respectivamente. $\mathrm{El}$ algoritmo $\mathrm{OCx}$ es una relación polinómica de cuarto orden entre una relación de $R_{r s} y$ CCA.

$$
\log _{10}(\mathrm{CCA})=\mathrm{a}_{0}+\sum_{\mathrm{i}=1}^{4} \mathrm{a}_{\mathrm{i}}\left(\log _{10}\left(\frac{\mathrm{R}_{\mathrm{rs}}\left(\lambda_{\text {azul }}\right)}{\mathrm{R}_{\mathrm{rs}}\left(\lambda_{\text {verde }}\right)}\right)\right)^{\mathrm{i}},
$$

donde el numerador, $\mathrm{R}_{\mathrm{rs}}\left(\lambda_{\mathrm{azul}}\right)$, es el mayor de varios valores de entrada $R_{r s} y$ los coeficientes, ${ }^{a} 0-a_{4}$, para MODIS son derivados usando la base de datos versión 2 de NOMAD (NASA bio-Optical Marine Algorithm Data Set). Para estimaciones de CCA por debajo de $0.15 \mathrm{mg} \mathrm{m}^{-3}$, se utiliza el algoritmo CI. Para CCA superior a $0.2 \mathrm{mg} \mathrm{m}^{-3}$, se utiliza el algoritmo OCx. Mientras para valores entre 0.15 y $0.2 \mathrm{mg} \mathrm{m}^{-3}$, los algoritmos CI y OCx se combinan utilizando un enfoque ponderado. Los mosaicos diarios de la clorofila MODIS que cubren el área de estudio de extensión NetCDF, con resolución espacial de $1 \mathrm{~km}$ son recolectados desde la página web de la NASA (http://oceancolor.gsfc.nasa.gov), para el periodo de 01 de enero de 2008 a 31 de diciembre de 2015.

El área de estudio está conformada por las bahías de Sechura y Paita, delimitados entre las longitudes de $83^{\circ}$ $80^{\circ} \mathrm{O}$ y latitudes de $3^{\circ}-7^{\circ} \mathrm{S}$ al noroeste del Perú. El área de estudio está ubicada en la zona de convergencia de las aguas frías de la Corriente Costera Peruana (CCP) y las Aguas Ecuatoriales Superficiales (AES) cálidas. La bahía de Sechura en general es alimentada por aguas costeras frías $\left(16^{\circ} \mathrm{C}-20^{\circ} \mathrm{C}\right)$ provenientes del sur, con relativamente altas en salinidad que varían en un intervalo de 34.9 a 35.0 ups (unidad practica de salinidad), aunque en verano austral la proyección de las AES $\left(20^{\circ} \mathrm{C}-24^{\circ} \mathrm{C}\right.$ y $34.0-$ 34.8 ups) se encuentran al norte de Paita, donde se registran mezclas entre las aguas afloradas debido a la convergencia de las dos masas de agua, registrándose como consecuencia en su superficie los valores más bajas de salinidad (Morón et al., 2013). Su borde costero de la bahía de Sechura se caracteriza por la presencia del estuario de Virrillá, los manglares, plantas de harina y aceite de pescado (Copeinca y Perupez).

\section{Metodología}

Se implementó el plugin EPOC (ENVI Plugin for Ocean Color) en ENVI/IDL en el sistema operativo W10 para pre-procesar y re-proyectar los datos imágenes diarios de nivel 2 correspondiente a TSM y la CCA. Los datos de TSM diurnos y la CCA utilizados para el promedio climatológico son re-proyectadas usando la proyección geográfica con Datum WGS84 en formato Geo TIFF. Para buena calidad de datos imágenes es aplicado a TSM la 
máscara de calidad qual_sst considerando los niveles de calidad 0,1 y 2 en el lenguaje de programación IDL. En nuestro caso los pixeles con niveles de calidad 3 y 4 no son considerados como datos válidos. El promedio climatológico es obtenido como el promedio de datos imágenes diarios de nivel 2 de MODIS/Aqua (01 de enero 2008 al 31 de diciembre de 2015). La validación de datos mensuales de TSM satelital en la estación Paita es evaluada mediante la regresión lineal simple. Como bondad de ajuste del modelo se ha utilizado el coeficiente de correlación de Pearson y medida del error el RMSE (error cuadrático medio). Los promedios mensuales de TSM MODIS utilizados para la validación y anomalía interanual son de tipo SMI (Standard Mapped Image) y tienen una resolución espacial de $4 \mathrm{~km} \mathrm{x} 4 \mathrm{~km}$. La anomalía interanual estandarizada se obtiene como la diferencia entre el valor promedio anual y el promedio general de datos mensuales para toda el área de estudio dividido entre su desviación estándar de todos los datos mensuales, el cual puede ser positivo o negativo.

\section{Resultados y Discusión}

El parámetro temperatura tiene una gran influencia en el comportamiento de la atmosfera y el clima (Brown et. al., 1999). El TSM es sensible a la cantidad de calor proveniente de tres fuentes: calor del interior de la Tierra, calor de degradación radiactiva y calor por radiación solar. Los elementos tomados en cuenta para la radiación solar son la radiación solar directa y la difusa. Los factores naturales que inducen en el cambio de la temperatura son: circulación atmosférica (movimiento del aire a gran escala), corrientes marinas, latitud (tiempo de insolación), topografía costera-subacuática y profundidad del océano. La información referente a la distribución de la temperatura es específicamente aplicada a la localización de corrientes marinas, frentes térmicos y entre otros (Rojas y Eche, 2006). En el mapa TSM climatológico, representado en la Figura 1a, se observa el contraste del efecto de la convergencia de la corriente Humboldt y de aguas cálidas superficiales procedentes del ecuador (Yupanqui et al., 2007). La temperatura en proximidades a punta Aguja y Gobernador muestran un incremento hacia el noroeste, siendo la temperatura menor cerca de la costa que la temperatura en mar adentro. Es de destacar en relación a la bahía Sechura una angosta franja de aguas relativamente caliente próximo a línea costera, cuyos valores de TSM varían en promedio entre $21^{\circ} \mathrm{C}$ y $22^{\circ} \mathrm{C}$. $\mathrm{La}$ condición media de TSM en la bahía de Sechura y Paita oscila entre los $19.5^{\circ} \mathrm{C}$ y $22^{\circ} \mathrm{C}$, desviación típica de $0.26^{\circ} \mathrm{C}$. El histograma de frecuencia de la TSM mostrado en la Figura $1 b$ corresponde a promedio climatológico de la bahía de Sechura. Se observa que la distribución de frecuencia es sesgada hacia derecha (distribución bimodal). La estadística correspondiente a la bahía Sechura (polígono sombreado de color rojo, ubicado en la esquina superior derecho), muestra la máxima $\mathrm{TSM}$ de $21.83^{\circ} \mathrm{C}$ al noroeste y mínima $\left(20.32^{\circ} \mathrm{C}\right)$ al sur de la bahía, la media de $20.70^{\circ} \mathrm{C}$ con desviación estándar de $0.27^{\circ} \mathrm{C}$ de 1034 pixeles válidos.

La información (bandas 31 y 32) registrada por MODIS en la región infrarroja térmica (entre 10 y $12 \mu \mathrm{m}$ ) es utilizada para estimar la TSM diurna. Las bandas 31 y 32 de MODIS son próximos a la longitud de onda a la cual el cuerpo negro tiene su emisión máxima de energía (una aproximación a la temperatura media de la Tierra). Además, las bandas referidas son más anchas y no presentan problemas de reflexión especular (Brown et al., 1999). En la Figura 2a, se observa el diagrama de dispersión de TSM in-situ y Satelital con coeficiente de correlación de 97\%. Los datos de temperatura "in-situ" son recogidos de la capa de mezcla, "Bulk". El significado "Bulk" de acuerdo a los científicos oceanógrafos es la medida de la TSM registrados por barcos o boyas con sensores de temperatura sumergidos a una pequeña profundidad, que varían desde centímetros a metros. Mientras la temperatura satelital estimada de los sensores de radiación infrarrojo-térmica, detectan la temperatura radiativa emitida por una delgada capa superficial, "la piel del mar", cuyo espesor apenas puede llegar a alcanzar un milímetro. Asimismo los promedios mensuales de TSM insitu registrados en la estación de Paita (1998-2015) presenta un valor máximo superior de $29.34^{\circ} \mathrm{C}$ para el mes de febrero del año 1998 y $14.84{ }^{\circ} \mathrm{C}$ (mínimo) para octubre de 2010. Los eventos cálidos de 2014-2015 presentó una anomalía estandarizada de +0.65 , en el análisis de la climatología interanual (Figura 2b).

La bahía de Sechura tiene una correlación positiva significativa ( $\mathrm{R}=70.4 \%)$ entre la TSM y la CCA (Figura 3 ), ya que al aumentar la temperatura esta influye en el incremento de la CCA de la zona. Esto sugiere que la correlación entre la clorofila-a y TSM no siempre es negativa como ocurre en las aguas del archipiélago de Spermonde (sur de China) y bahía de Bengal (India). La correlación positiva para la bahía (Figura 3) es posiblemente debido al proceso de afloramiento marino ocurrido por la corriente Humboldt y estrés del viento (vientos costeros muy fuertes en invierno).

\section{Conclusiones}

La TSM-MODIS mensuales y la TSM in-situ mensuales en la bahía de Paita presenta una correlación de $(\mathrm{R}=96 \%)$, por lo que podemos plantear su uso en modelos de variables oceanográficos. Existe aproximadamente una relación lineal entre TSM y CCA a partir del sensor VIIRS, lo cual no está de acuerdo con la literatura mundial sobre ambos en otros lugares.

\section{Agradecimientos}


Rev. Inv. Fís. 23(3), (2020)

Los autores agradecen a CONCYTEC por el soporte financiero a través de su Programa de Centros de
Excelencia, el cual ha hecho posible el desarrollo de este trabajo de investigación.

\section{Referencias}

Brown O. y Minnett P. (1999). MODIS infrared sea surface temperature algorithm - Algorithm Theoretical Basis Document Version 2.0. University of Miami. https://modis.gsfc.nasa.gov/data/atbd/atbd_mod25.pdf.

Hu, C., Lee, Z., y Franz, B. (2012). Chlorophyll algorithms for oligotrophic oceans: A novel approach based on threeband reflectance difference. Journal of Geophysical Research: Oceans, 117(C1).

Morón O., Velazco F. y Beltrán L. (2013). Características hidrográficas y sedimentológicas de la bahía de Sechura. Instituto del Mar del Perú - Informe No. 40(3-4):150-9.
Rojas J. y Eche J. (2006). La temperatura de la superficie del mar peruano a partir de las imágenes AVHRR / NOAA (2000-2003). Revista de Investigación de Física de la Universidad Nacional de Mayor de San Marcos - Perú. Vol. 9 No. 1, pp 24-30.

Walton C., Pichel W., Sapper J. y May, D. (1998). The development and operational application of nonlinear algorithms for the measurement of sea surface temperatures with the NOAA polar-orbiting environmental satellites. Journal of Geophysical Research: Oceans, 103, 27999-28012.

O'Reilly, J. (2000). Ocean color chlorophyll-a algorithms for SeaWiFS, OC2, and OC4: Version 4. SeaWiFS Postlaunch Calibration and Validation Analyses. 11. 9-23.

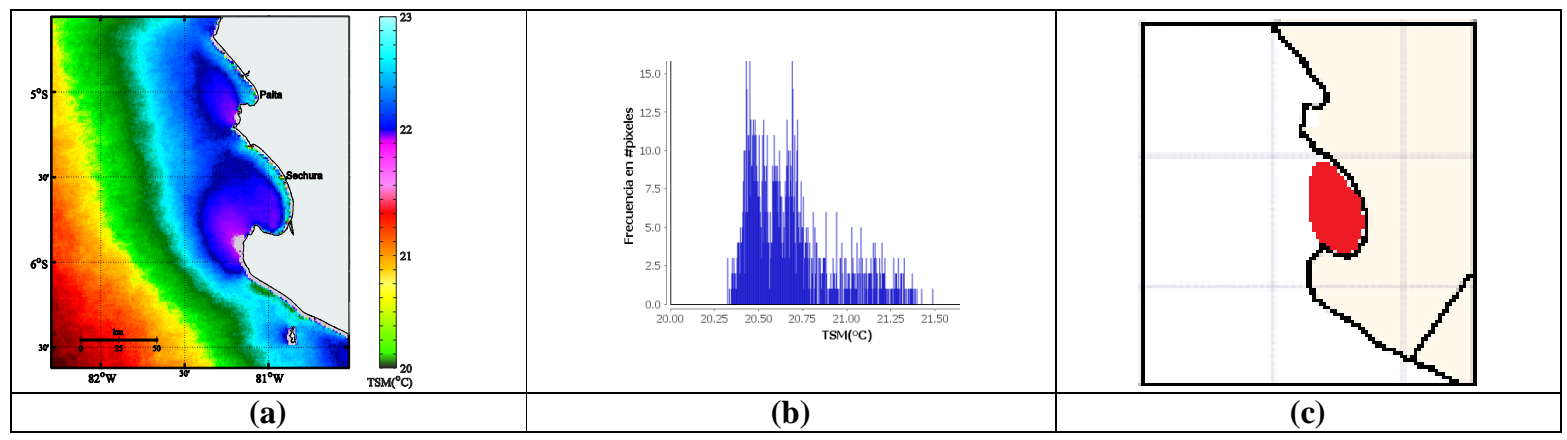

Figura 1. (a) Promedio climatológico de TSM para el periodo 2008-2015, obtenido con datos diarios del sensor MODIS/AQUA. (b) Histograma de número de ocurrencias de TSM promedio (2008-2015) para la "bahía de Sechura" (c) área de estudio.

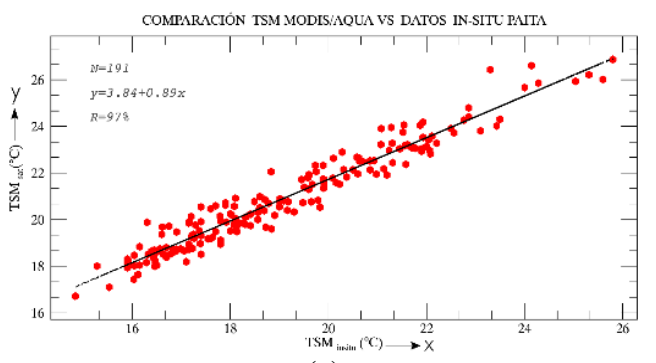

(a)

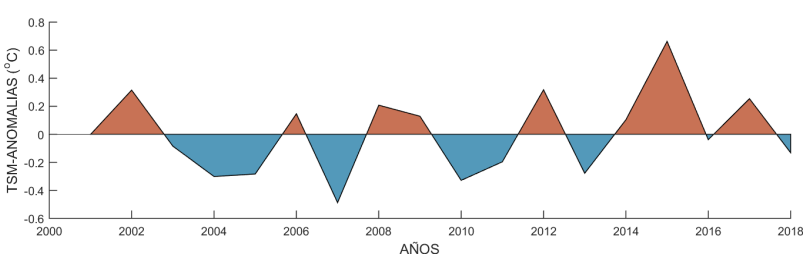

(b)

Figura 2. (a). Diagrama de dispersión para TSM promedio mensual estimada desde datos imágenes (MODIS y VIIRS) e insitu registrada en la estación Paita (ubicada en la parte sur de la bahía) por IMARPE, periodo de 2000 a 2015. (b) Promedios anuales de las anomalías de la TSM para la estación Paita, procesado con datos imágenes del sensor VIIRS. 
Rev. Inv. Fís. 23(3), (2020)

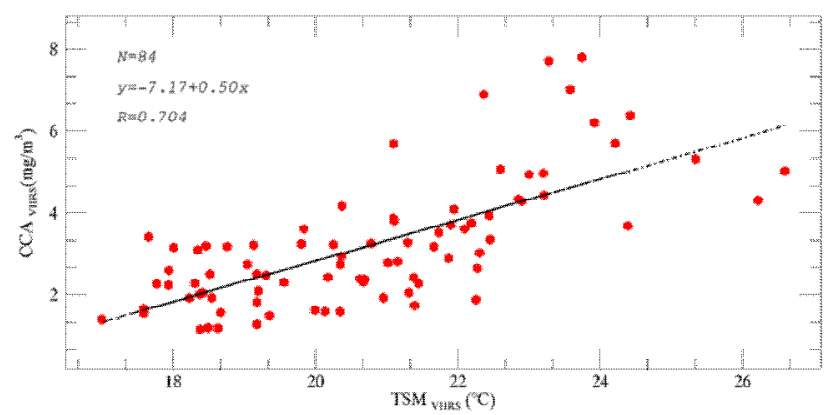

Figura 3. Relación entre la CCA y TSM para un conjunto de 84 pares de datos mensuales pertenecientes a la bahía de Sechura. Los promedios mensuales de TSM y CCA corresponden a los productos del sensor VIIRS, periodo de 2012 a 2018. 